started a new IV chemotherapy regimen a month before death. About lines of treatment, $45.25 \%$ (38) of the patients received firstline chemotherapy, $20.25 \%$ (17) in second line, $21.4 \%$ (18) in third line and $13.1 \%$ (11) received more than 3 lines of chemotherapy. In $48.75 \%$ (39), the percentage of the last dose of IV chemotherapy administered was $\leq 80 \%$. All patients were admitted to the Oncology floor at some point in the last 30 days of life, with an average stay of 9.73 days.

Conclusions The percentage of patients receiving IV chemotherapy in the last 14 days of life and that of those who started with a new regimen a month before death are much higher in our hospital than in similar studies. In view of the results obtained, more than half of these patients received IV chemotherapy in the last month of life. This makes us ask ourselves what factors contributed to this decision to treat, were the benefit and toxicity correctly assessed and was it is really necessary to have active cancer treatment in the last days of life?

No conflict of interest.

\section{OHP-081 USE OF LOW THERAPEUTIC UTILITY DRUGS IN AN INSTITUTION BEFORE THEIR USE WAS RESTRICTED IN THE SPANISH HEALTH SYSTEM}

doi:10.1136/ejhpharm-2013-000276.454

B Arribas-Díaz, A Bosó-Ribelles, MA Moregó-Soler, MC Sánchez-Mulero, M TobaruelaSoto, P Selvi-Sabater, AM Rizo-Cerdá, MM Sánchez-Catalicio, MP Molina-Guillén, N Ramón-Manresa. Hospital Morales Meseguer, Hospital Pharmacy, Murcia, Spain

Background Low therapeutic utility drugs (LTUDs) are those with controversial efficacy that provide little improvement for the disease or the symptoms.

These drugs have recently been removed from the system financing Spanish healthcare, with the aim of controlling healthcare expenditure.

Purpose To assess the use of these drugs in institutionalised older people and find out how the new law may be affecting it.

Materials and Methods This was a retrospective transversal study. We choose one day at random and checked all treatments prescribed that day.

The following data were collected: drugs, sex, age and LTUDs.

The data were obtained from the SAVAC programme and processed in Excel.

Results A total of 175 residents were included, mean age 89 years old.

LTUDs were administered to 65 people (37\%).

There were 1812 different drugs, of which 88 (4.9\%) were LTUDs, measured as number of dosage units.

Drug consumption in primary care $(\mathrm{PC})$ is measured by number of packs, not as number of dosage units. During the study, PC consumption of LTUD accounted for $6.86 \%$ of the total.

The LTUDs prescribed were: 26 items (30.3\%) acetylcysteine, 18 $(21.5 \%)$ topical diclofenac, $12(14.4 \%)$ citicoline, 10 (12.0\%) trimetazidine, 9 (10.8\%) pentoxifylline, 4 (4.9\%) piracetam, $2(2.5 \%)$ ambroxol, 1 (1.2\%) acetaminophen plus codeine, $1(1.2 \%)$ escine and $1(1.2 \%)$ inhaled mesna.

Conclusions Institutionalized older people use fewer LTUDs than patients from PC.

Mucolytic agents and topical NSAIDs are on top of the list, accounting for $50 \%$ of the LTUDs used.

Nearly $40 \%$ of institutionalised people will have to pay for these $5 \%$ of their drugs, or these medicines will have to be removed from their treatments.

Better designed studies should be done to clarify the real efficacy and efficiency of this large group of drugs.

No conflict of interest.

\section{OHP-082 USE OF STANDARD PROTOCOLS FOR TOTAL PARENTERAL NUTRITION IN A TERTIARY UNIVERSITY HOSPITAL}

doi:10.1136/ejhpharm-2013-000276.455

P Carmona Oyaga, C Ripa Ciaurriz, B Odriozola Cincunegui, MJ Gayan Lera, M Ercilla Liceaga, N Mauelon Echeverria, K Andueza Granados, P Pascual Gonzalez, J Barral Juez, M Umerez Igartua. Donostia University Hospital, Pharmacy Service, San Sebastián, Spain

Background One of the clinical pharmacist's main functions in parenteral nutrition is to ensure the quality and safety of the solutions prepared. It is too laborious to do this with each preparation. So in our hospital it was decided to design 21 standard Total Parenteral Nutrition (TPN) protocols.

Purpose To analyse the prescriptions for TPN and their compliance with the standard protocols available.

Materials and Methods A retrospective study was conducted over a period of one year (October 2011-October 2012). The composition of all TPN administered to adults was recorded, as well as the addition of various drugs such as insulin or somatostatin. Data were obtained from the pharmacy service's nutritional database.

Results 629 adult patients were treated with TPN and received 8342 bags of TPN; 3129 (37.5\%) fitted the standard protocols. The changes in the composition of TPN in non-standard TPN bags were: glucose added to 117 (2.3\%) bags, lipids in quality $2276(44.4 \%)$ and in quantity $374(7.5 \%)$, nitrogen to $223(4.3 \%)$; electrolytes: sodium to $238(4.6 \%)$, calcium to $7(0.1 \%)$, magnesium to 181 (3.5\%), potassium to 3054 (59.6\%) and phosphorus to 245 (4.8\%); volume to 117 (2.3\%), somatostatin to $545(10.6 \%)$ and insulin to $862(16.8 \%)$.

Composition of protocols ranged from: nitrogen: 6 to $20 \mathrm{~g}$, increasing the amount of nitrogen from 2 by 2 g, glucose: 150-200250-300 g, lipids 0-50-75-100 g, kcal non-protein/g nitrogen from 87.5 to 187.5 and volume $1350-2000-3000 \mathrm{~mL}$. All protocols contained the same amount of electrolytes (sodium: $75 \mathrm{mEq}$, potassium: $60 \mathrm{mEq}$, calcium: $15 \mathrm{mEq}$, magnesium: $15 \mathrm{mEq}$, chloride: $90 \mathrm{mEq}$, acetate: $75 \mathrm{mEq}$ and phosphorus: $10-20 \mathrm{mMol}$ ), vitamins and trace elements.

Conclusions $61 \%$ of administered TPN needed to be modified with respect to standard protocols in order to meet the nutritional requirements of individual patients. So we are considering revising the protocols regarding the quality of lipids and amount of potassium.

No conflict of interest.

\section{OHP-083 USTEKINUMAB FOR THE TREATMENT OF PSORIASIS IN A TERTIARY HOSPITAL}

doi:10.1136/ejhpharm-2013-000276.456

E Ramió, I Javier, N El Hilali Maso, Gl Ballesteros, M Pons, M Aguas. Capio Hospital Universitari Sagrat Cor, Pharmacy, Barcelona, Spain

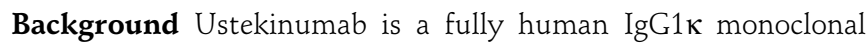
antibody against interleukin 12 and 23 indicated for the treatment of moderate to severe plaque psoriasis in adults who have failed to respond to previous treatment. The recommended posology is an initial dose of $45 \mathrm{mg}$ ( $90 \mathrm{mg}$ with a body weight $>100 \mathrm{Kg}$ ) subcutaneously, followed by the same dose 4 weeks later, and then every 12 weeks thereafter.

Purpose To analyse the use of ustekinumab in our hospital since its launch.

Materials and Methods Retrospective longitudinal study of all the patients with psoriasis treated with ustekinumab since its launch in January 2009 in a tertiary hospital. Data was obtained from the records of outpatients who get their medicines from the hospital pharmacy, and before February 2010, we used records of 
ustekinumab prescriptions that required validation from the inspection service.

Main outcome measures: gender, age, dose, time in treatment, previous use of a biological antiTNF, changes in frequency of administration, induction posology at the beginning.

Statistics: Descriptive analysis of qualitative and quantitative data, unpaired t-test with SPSS 17.0.

Results The review consisted of 57 patients (56.1\% men) with a mean age of 52 (SD 12.9) for men and 43 years old (SD 19.2) for women $(\mathrm{P}=0.05)$. In 9 patients (one woman) the dose used was $90 \mathrm{mg}$. In 35 cases $(61.4 \%)$ the patients received a previous treatment with biological antiTNF and in 12 patients the treatment started every 12 weeks directly (without induction). The physicians changed the frequency in 10 patients (17.5\%): 5 with doses every 16-20 weeks and 5 with interruptions with a mean of 7.6 months.

Currently 11 patients have stopped the treatment and the average time of treatment is 19.3 months (SD 9.9).

Conclusions Ustekinumab was the first-line biological treatment in $38.6 \%$ of patients.

A significant number of patients used $90 \mathrm{mg}$, and it could be interesting to evaluate whether a $45 \mathrm{mg}$ dose would be sufficiently effective to reduce the cost.

No conflict of interest.

\section{OHP-084 WHAT CONCEPTS ARE USED TO DESCRIBE THE COOPERATION MECHANISMS BETWEEN THE HOSPITAL SECTOR AND PRIMARY CARE? ANALYSIS OF TERMINOLOGY}

doi:10.1136/ejhpharm-2013-000276.457

'S Vogler, 'N Zimmermann, ${ }^{1} \mathrm{C}$ Habl, ${ }^{1} \mathrm{C}$ Leopold, ${ }^{1} \mathrm{~K}$ Habimana, ${ }^{2} \mathrm{~A}$ Mantel-

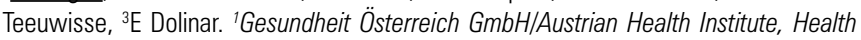
Economics, Vienna, Austria; 2 Utrecht University, WHO Collaborating Centre for Pharmacoepidemiology and Pharmaceutical Policy Analysis, Utrecht, The Netherlands; ${ }^{3}$ Retired, Chief Hospital Pharmacist, Vienna, Austria

Background Cooperation between the hospital sector and primary care is addressed under different names which hampers sharing and identifying existing practises and policies in this field.

Purpose To get a better understanding of the concept of medicines management at the interface of the hospital and primary care sectors (hereafter called interface management).

Materials and Methods Narrative literature review searching Medline, EMBASE, GoogleScholar, Web of Science (ISI), supplemented by hand searching (snowballing) to detect grey literature and contacts with policy makers, researchers and hospital pharmacists to identify further references. Search terms included interface (management), seamless care, continuous care, transitional care, transition in combination with medication, medicines, drugs and pharmaceuticals. Interventions that did not address medicines were excluded; the search period was 1990 to September 2012.

Results In English-language literature, the most commonly applied terms are seamless care, integrated care, comprehensive care, transmural care, transitional care and continuity of care for which, in most cases, generally accepted and repeatedly quoted definitions exist. A more recent terminology is 'interface management'. In many cases, specific projects such as hospital discharge programmes are described without any explicit reference to overall concepts such as interface management or seamless care. Tools such as medicines reconciliation and/or patient counselling can be used to improve medicines management at the interface but they are not necessarily used as specific interface management measures.

Conclusions Even in the English-language literature, the mechanisms of cooperation between the hospital sector and the primary care are referred to under different names. It is recommended to include specific interface management measures as search terms in a literature review on interface management since overall concepts such as seamless care and interface management are likely to yield few results. Terminology work to increase clarity in this field is needed.

No conflict of interest.

\section{Clinical pharmacy and clinical trials (including case series)}

\section{CPC-001 A CLINICAL PHARMACIST FOR OUTPATIENT CONSULTATIONS IN A HEART FAILURE CLINIC}

doi:10.1136/ejhpharm-2013-000276.458

'0 Jullion, ${ }^{2 P}$ Sterckx, ${ }^{2} \mathrm{~S}$ Huez, ${ }^{2} \mathrm{JL}$ Vachiery, ${ }^{1} \mathrm{~S}$ Lorent. 'Erasme Hospital, Pharmacy, Brussels, Belgium; '2Erasme Hospital, Cardiology, Brussels, Belgium

Background Heart Failure (HF) is a severe chronic condition requiring polymedication, which is associated with a risk of nonadherence to chronic Heart Failure (CHF) treatment.

We recently demonstrated that a clinical pharmacist (CP) can be successfully integrated into a cardiology department to improve HF patient care by supervising the treatment. In addition, in 2009 we developed a dedicated CP outpatient consultation integrated in the Heart Failure Clinic (HFC) at our institution.

Purpose To provide a description of the role of the CP in an outpatient HFC.

Materials and Methods 325 patients with HF are monitored at our HFC. All patients are seen by the CP during a dedicated consultation, which includes the following: 1) preparation of the patient's file; 2) the complete history of medical treatment is checked; 3) the record of drugs is updated; 4) vital signs and electrocardiography are performed by the HF nurse. The patient is then seen by the cardiologist who updates the treatment plan. The patient is then seen by the $\mathrm{CP}$ who draws up and issues a plan to put the new treatment into practise, which includes scheduling phone contact for drug uptitration. Finally, the CP and the HF nurse ensure that the plan is followed by weekly scheduled phone consultations.

Results Each HF patient is seen at the outpatient clinic for approximately 45 minutes. On a yearly basis, the CP establishes 584 pharmaceutical plans and performs 197 phone consultations for follow up, resulting in 97 changed treatments.

Conclusions The integration of a CP into the HFC is important to improve management of HF through dedicated outpatient consultations, implementation of treatment plans and checking the patient's adherence.

No conflict of interest.

\section{CPC-002 A MULTICENTRE RETROSPECTIVE STUDY TO EVALUATE THE ECONOMIC IMPACT OF THE PRESCRIBING MODELS FOR TRASTUZUMAB IN THE PIEMONTE REGION}

doi:10.1136/ejhpharm-2013-000276.459

${ }^{1} \mathrm{DA}$ Barilà, ${ }^{1} \mathrm{~A}$ Bianco, ${ }^{2} \mathrm{~S}$ Bustreo, ${ }^{2} \mathrm{M}$ Donadio, ${ }^{3} \mathrm{~S}$ Rosso, ${ }^{1} \mathrm{~F}$ Cattel. ${ }^{1} \mathrm{~A} .0$ Città della Salute e della Scienza di Torino, S.C Pharmacy, Turin, Italy; ${ }^{2}$ A. O Città della Salute e della Scienza di Torino, Medical Oncology Senological Breast Unit, Turin, Italy; ${ }^{3} A .0$ Città della Salute e della Scienza di Torino, CPO Piedmunt, Turin, Italy

Background In recent years, there has been a rapid and constant increase in the costs of cancer treatment but, with limited health care resources, it is essential to consider the economic implications of different health interventions. 